\title{
AVALIAÇÃO DA LINGUAGEM ORAL E ESCRITA EM SUJEITOS COM SÍNDROME DE ASPERGER
}

\section{Language assessment in subjects with Asperger Syndrome}

\author{
Karin Ziliotto Dias ${ }^{(1)}$, Renata Cristina Dias da Silva ${ }^{(2)}$, Liliane Desgualdo Pereira ${ }^{(3)}$, \\ Jacy Perissinoto ${ }^{(4)}$, Carla de Queiroz Bergamini ${ }^{(5)}$
}

\section{RESUMO}

Objetivo: avaliar e caracterizar provas fonoaudiológicas de linguagem oral e escrita de sujeitos com Síndrome de Asperger comparativamente a um grupo de sujeitos com desenvolvimento típico. Métodos: avaliou-se 44 sujeitos que constituíram dois grupos: o grupo Asperger, composto por 22 sujeitos diagnosticados por equipe multidisciplinar como portadores de Síndrome de Asperger, conforme os critérios do DSM-IV; e o grupo de comparação, denominado grupo de baixo risco para alterações do desenvolvimento, também com 22 participantes, pareados com os sujeitos do grupo Asperger segundo a idade cronológica. Todos os sujeitos eram do sexo masculino, com idade cronológica entre 10 e 30 anos e quociente intelectual maior ou igual a 68 e foram submetidos à Prova de Consciência Fonológica, Teste de Vocabulário por Imagem Peabody, Prova de Leitura de Palavras e Pseudopalavras, Prova de Compreensão de Leitura, Prova de Escrita sob Ditado de Palavras e Pseudopalavras, Prova de Escrita Semidirigida de Textos. Resultados: a análise estatística revelou diferenças estaticamente significantes entre as medianas da prova de consciência fonológica e entre as médias do teste de vocabulário por imagem Peabody e prova de compreensão de leitura nos dois grupos estudados $(p<0,05)$. Conclusão: na avaliação da linguagem oral e escrita, o grupo de sujeitos com Síndrome de Asperger caracterizou-se por um desempenho pior do que o grupo de sujeitos de baixo risco para alterações do desenvolvimento nas provas fonoaudiológicas de consciência fonológica, teste de vocabulário por imagem Peabody e prova de compreensão de leitura, o que caracteriza prejuízo em níveis fonológico, semântico e pragmático da linguagem.

DESCRITORES: Síndrome de Asperger; Transtornos da Linguagem; Testes de Linguagem

(1) Fonoaudióloga; Professora Adjunta Substituta do Curso de Fonoaudiologia da Universidade Federal de São Paulo; Doutora em Ciências pela Universidade Federal de São Paulo, UNIFESP, São Paulo, SP.

(2) Fonoaudióloga do Centro de Referência da Infância e Adolescência do Departamento de Psiquiatria da Universidade Federal de São Paulo; Mestre em Ciências pela Universidade Federal de São Paulo, UNIFESP, São Paulo, SP.

(3) Fonoaudióloga; Chefe do Departamento de Fonoaudiologia e Professora Adjunta do Curso de Fonoaudiologia da Universidade Federal de São Paulo; Doutora em Distúrbios da Comunicação Humana pela Universidade Federal de São Paulo, UNIFESP, São Paulo, SP.

(4) Fonoaudióloga; Professora Adjunta do Curso de Fonoaudiologia da Universidade Federal de São Paulo; Doutora em Distúrbios da Comunicação Humana pela Universidade Federal de São Paulo, UNIFESP, São Paulo, SP.

(5) Fonoaudióloga clínica do Núcleo de Estudos Fonoaudiológicos; Doutora em Ciências pelo Programa de Fisiopatologia Experimental da Faculdade de Medicina da Universidade de São Paulo, UNIFESP, São Paulo, SP.

Conflito de interesse: inexistente

\section{INTRODUÇÃO}

A Síndrome de Asperger ou "Psicopatia Autística" - como a síndrome foi inicialmente denominada pelo pediatra vienense Hans Asperger 1 que, em 1944 descreveu um grupo de crianças, principalmente meninos, com um padrão típico de comportamentos e déficits. Ao resumir as características típicas desta alteração, o autor pontuou a aparência física das crianças, seu bom nível intelectual, dificuldades de linguagem e de atenção, um comportamento problemático em situações sociais e dificuldades emocionais.

Em 1981, seus estudos originais foram revistos e algumas modificações em relação ao conceito original foram sugeridas ${ }^{2}$. Foi, então, proposta a utilização do termo Síndrome de Asperger e a ideia de que existe um espectro de desordens autistas que 
inclui sujeitos com diferentes níveis de inteligência e linguagem, mas com uma tríade de dificuldades nas áreas de interação social, comunicação e jogo simbólico ${ }^{2}$.

Há mais de dez anos, o DSM Diagnostic and Statistical Manual of Mental Disorders -IV ${ }^{3}$ e o CID Classificação Estatística Internacional de Doenças e Problemas Relacionados à Saúde $-10{ }^{4}$ compilaram os critérios diagnósticos para a Síndrome de Asperger, nos quais foram mencionados prejuízos de interação social, comunicação e imaginação, interesses restritos, pensamento concreto e literal e padrões estereotipados de comportamentos, sem importantes atrasos cognitivos e de linguagem oral, aparentemente fazendo referência apenas à presença da fala no final do primeiro ano de vida.

Embora os sujeitos com síndrome de Asperger não apresentem atrasos importantes na área da linguagem, inclusive muitos deles tem uma habilidade para expressar-se verbalmente de maneira criativa, utilizando vocabulário sofisticado e estruturas gramaticais corretas, nota-se uma dificuldade em utilizar a linguagem efetivamente nas situações cotidianas. Há relatos na literatura de dificuldades quanto à prosódia ${ }^{2}$ e alterações em níveis semânticos e pragmáticos da linguagem ${ }^{5-9}$. Recentemente, poucos estudos foram publicados com o objetivo de avaliar a linguagem do ponto de vista fonológico, sintático e semântico em sujeitos com Síndrome de Asperger e idade superior a 10 anos.

Para compreender melhor a linguagem em sujeitos com Síndrome de Asperger, o objetivo deste estudo é avaliar e caracterizar provas fonoaudiológicas de linguagem oral e escrita em sujeitos com Síndrome de Asperger comparativamente a um grupo de sujeitos com desenvolvimento típico.

\section{MÉTODOS}

Este estudo foi realizado no Departamento de Fonoaudiologia da Universidade Federal de São Paulo - Escola Paulista de Medicina. Todos os pacientes foram informados oralmente e por meio de material escrito a respeito dos objetivos da pesquisa e dos procedimentos a serem realizados. Os participantes assinaram termo de consentimento e esclarecimento antes do início do estudo.

Nesta pesquisa, foram avaliados 44 sujeitos que constituíram dois grupos. O grupo Síndrome de Asperger, abreviadamente denominado GA, foi composto de 22 sujeitos diagnosticados por equipe multidisciplinar como portadores de Transtorno Global do Desenvolvimento do subtipo Síndrome de Asperger. E, o grupo de comparação, denominado grupo de baixo risco para alterações do desenvolvimento - GBRAD, também foi composto de
22 participantes, pareados com os sujeitos do grupo GA segundo a idade cronológica.

A seguir, em tópicos, estão os critérios de inclusão dos participantes em cada um dos grupos estudados e a avaliação fonoaudiológica.

1. Diagnóstico médico de Síndrome de Asperger segundo os critérios do Manual Diagnóstico Estatístico de Transtornos Mentais - IV ${ }^{3} \mathrm{e}$ ausência de outras condições mórbidas psiquiátricas. Importante ressaltar que, neste estudo, todos os sujeitos avaliados já apresentavam o diagnóstico de Síndrome de Asperger em sua instituição de origem, conforme os critérios do DSM-IV 3 ;

2. Diagnóstico psicológico do nível de quociente intelectual estimado maior ou igual a 68 $(\geq 68)$, segundo a Escala Wechsler ${ }^{10}$; WISC Wechsler Intelligence Scale for Children e WAISWechsler Adult Intelligence Scale;

3. Idade cronológica entre 10 anos e 30 anos;

4. Sexo masculino;

5. Preferência manual direita, conforme os resultados do questionário de Edinburgh modificado ${ }^{11}$;

6. Ausência de evidência de deficiências físicas;

7. Avaliação audiológica dentro dos critérios de normalidade estabelecidos. O critério de limiar de audibilidade normal utilizado foi a presença de níveis de audição inferiores a 25dBNA, Padrão ANSI - 1969, em todas as frequências sonoras avaliadas ${ }^{12}$.

Para a formação do grupo GBRAD foram considerados todos os itens descritos acima exceto o item 1.

A avaliação da linguagem oral e escrita foi realizada por uma fonoaudióloga da Universidade Federal de São Paulo, com experiência no uso e interpretação dos procedimentos de avaliação utilizados. Os procedimentos foram filmados em fita VHS com filmadora Panasonic Palmcorder NV-RJ30PN, gravados em fita k-7 com gravador AIWA TP-VS485 Voice Sensor.

Para a avaliação da linguagem oral e escrita, um conjunto de testes foi eleito contemplando as seguintes provas:

\section{Prova de Consciência Fonológica (PCF)}

A prova de consciência fonológica desenvolvida e utilizada ${ }^{13}$ para avaliar a habilidade de sujeitos em manipular os sons da fala. Seu resultado é um escore máximo de 40 pontos, pois é composto por dez subtestes, cada um com quatro itens.

Segue abaixo, uma descrição de cada um dos dez subtestes (provas-P):

1. P1 - Síntese Silábica: o examinador fala as sílabas de uma palavra com um intervalo de 
um segundo entre elas e o sujeito deve uni-las, dizendo qual palavra resultou da união.

2. P2 - Síntese Fonêmica: como na tarefa anterior, o examinador fala os fonemas de uma palavra separados e o sujeito deve uni-los, dizendo a palavra resultante da união.

3. P3 - Rima: o sujeito deve selecionar dentre três palavras, as duas que terminam com o mesmo som.

4. P4 - Aliteração: o sujeito deve selecionar dentre três palavras, as duas que começam com o mesmo som.

5. P5 - Segmentação Silábica: o sujeito deve repetir a palavra dita pelo examinador, mas separada nas suas sílabas componentes.

6. P6 - Segmentação Fonêmica: o sujeito deve repetir a palavra dita pelo examinador, mas separada nos seus fonemas componentes.

7. P7 - Manipulação Silábica: o sujeito deve adicionar e subtrair sílabas de palavras, dizendo qual a palavra formada.

8. P8 - Manipulação Fonêmica: o sujeito deve adicionar e subtrair fonemas de palavras, dizendo qual a palavra formada.

9. P9 - Transposição Silábica: o sujeito deve inverter a ordem das sílabas de palavras, dizendo qual a palavra formada.

10.P10 - Transposição Fonêmica: o sujeito deve inverter a ordem dos fonemas de palavras, dizendo qual a palavra formada.

\section{Teste de Vocabulário por Imagem Peabody (TVIP) \\ O Teste de Vocabulário por Imagem Peabody} (TVIP) ${ }^{14}$, é um teste de habilidade verbal, criado para medir o vocabulário receptivo.

O TVIP foi utilizado para avaliar o vocabulário receptivo e consiste de 125 palavras e 125 pranchas com quatro desenhos cada uma, em branco e preto. Os desenhos contemplam várias categorias: pessoas, ações, qualidades, partes do corpo, tempo, natureza, lugares, objetos, animais, termos matemáticos, ferramentas e instrumentos. $\mathrm{O}$ examinador fala uma palavra e o examinado deve apontar o único desenho que representa a palavra falada. $O$ procedimento para a realização deste teste seguiu as recomendações dos autores.

A análise dos resultados do teste foi feita levando-se em conta o número absoluto de acertos (método da pontuação simples).

\section{Leitura}

Prova de Leitura de Palavras e Pseudopalavras (PLPP)

Esta prova foi desenvolvida para avaliar leitura, foi utilizada conforme preconizado por seus autores ${ }^{15}$
A prova é composta por 90 itens que são lidos em voz alta pelo indivíduo e contém palavras tanto de alta como de baixa frequência, distribuídas em palavras regulares, irregulares e regras, com duas ou três sílabas e contém também pseudopalavras. O resultado nesta prova é apresentado em número de acertos. O máximo possível de acertos totaliza 90 pontos. Não foram analisados os tipos de erros cometidos pelos sujeitos.

\section{Prova de Compreensão de Leitura (PCL)}

Esta prova foi utilizada para avaliar a compreensão de leitura. Nesta prova, o examinador solicitou ao indivíduo que lesse um texto - "O urubu e as pombas" - em voz alta. Conforme proposto por sua autora ${ }^{16}$, a história é dividida em três episódios e 14 nodos principais. Verificou-se a compreensão do texto através da análise da recontagem do texto realizada pelo indivíduo. Sendo assim, a pontuação de cada sujeito no teste foi determinada pelo número de nodos recontados. A pontuação máxima obtida no teste é 14 acertos.

\section{$11^{\circ}$ Episódio}

1. Evento iniciador: continha uma ação: Um urubu ouviu dizer/

2. Cenário: uma oração: que na casa das pombas havia muita comida.

3. Reação: uma oração: Ele se pintou de branco.

4. Objetivo: uma oração: e voou até a casa das pombas.

5/6. Resultado: duas orações: As pombas acharam/que ele era uma delas

7. Final: uma oração: e o deixaram entrar.

$2^{\circ}$ Episódio

8. Evento iniciador: uma oração: Ele continuou a gritar como um urubu.

9. Reação: uma oração: As pombas descobriram/

10. Resultado: uma oração: que ele era um urubu/

11. Final: uma oração: e o expulsaram.

$3^{\circ}$ Episódio

12. Objetivo: uma oração: Ele tentou se juntar novamente aos urubus

13. Resultado: uma oração: mas estes não o reconheceram

14. Conclusão: uma oração: e não o aceitaram.

Os resultados desta prova foram classificados da seguinte maneira:

$0-3$ Nodos recontados

4-7 Nodos recontados

Não compreendeu

Compreendeu parcialmente

8 - 14 Nodos recontados 


\begin{abstract}
Escrita
Prova de Escrita sob ditado de palavras e pseudopalavras (PEPP)

Esta prova avaliou a escrita sob ditado de palavras e foi utilizado o procedimento proposto pelos autores ${ }^{15}$. O examinador lê em voz alta 72 palavras que devem ser escritas. Esta prova contém palavras de alta e baixa frequência e pseudopalavras, distribuídas em palavras regulares, irregulares e regras, com duas ou três sílabas.

Seu resultado é obtido como escore, ou número de acertos. O número máximo possível de acertos totaliza 72 pontos. Não foram analisados os tipos de erros cometidos pelos sujeitos.
\end{abstract}

Prova de Escrita Semi dirigida de Textos (PESDT)

Nesta prova, a figura inicial que compõe uma história é apresentada ao indivíduo e seu conteúdo Ihe é explicado ${ }^{17}$. Em seguida, outras três figuras da mesma história lhe são apresentas e lhe é solicitado que as ordene com o objetivo de sequencializar a história. Finalmente, o examinador solicita ao indivíduo que ele escreva um texto a partir da sequência de figuras montada.

O tipo de história utilizada para esta prova foi a "Comportamental II", ou seja, é um tipo de história que envolve pessoas engajadas em rotinas sociais e propiciam narrativas do tipo causal.

Para a análise da PESDT foi elaborado um sistema de pontuação para a história, segmentando-a e criando assim, um referencial quantitativo ${ }^{18}$. Portanto, a história - Comportamental II - denominada "O sorvete" foi assim dividida:

\begin{tabular}{|c|c|c|}
\hline Episódio 1 & Cenário & Nodo 1 \\
\hline \multirow[t]{2}{*}{ Episódio 2} & Ação 1: Menina pede sorvete & Nodo 2 \\
\hline & Ação 2: Menino não quer dar & Nodo 3 \\
\hline \multirow[t]{2}{*}{ Episódio 3} & Ação 1: Menina chega mais perto & Nodo 4 \\
\hline & Ação 2: Menino não quer dar & Nodo 5 \\
\hline \multirow[t]{2}{*}{ Episódio 4} & $\begin{array}{l}\text { Ação 1: Menina tira o sorvete } \\
\text { do menino }\end{array}$ & Nodo 6 \\
\hline & Ação 2: Menino chora & Nodo 7 \\
\hline
\end{tabular}

Cada ação escrita pelo sujeito, relativa à história, Ihe assegura um ponto. Se a ação ocorrida foi ignorada, ou foi narrada uma ação distinta da ocorrida, ele fica sem o ponto. Portanto, a pontuação de cada sujeito é determinada pelo número de nodos recontados por meio da escrita. O número máximo de acertos possíveis é sete pontos.

Os dados para este estudo foram coletados no período de abril de 2002 a setembro de 2004 . A coleta de dados foi realizada em duas sessões individuais, agendadas previamente com o paciente ou com os responsáveis, com duração média de 50 minutos cada sessão. A obtenção dos dados foi realizada sempre pelo mesmo profissional.
Este estudo foi aprovado pelo Comitê de Ética em Pesquisa da Universidade Federal de São Paulo / Hospital São Paulo, segundo as determinações do Conselho Nacional de Saúde (Resolução 196/96), conforme o protocolo CEP número 0341/02.

Para a análise descritiva, foram calculadas estatísticas descritivas (média, mediana, desvio-padrão, valores mínimo e máximo) com o objetivo de resumir os resultados obtidos para o grupo Síndrome de Asperger (GA) e para o grupo de baixo risco para alterações do desenvolvimento (GBRAD), considerando-se os critérios de inclusão de quociente intelectual estimado e os resultados das provas fonoaudiológicas.

Os resultados das provas fonoaudiológicas para a avaliação da linguagem oral e escrita nos grupos GA e GBRAD foram comparados considerando que o estudo seguiu um planejamento pareado. Nos casos em que não houve sérios desvios da normalidade, foi adotado o teste $\mathrm{t}-$ Student pareado na comparação das médias dos resultados das provas fonoaudiológicas nos dois grupos GA e GBRAD. Isso ocorreu para o TVIP, PLPP, PCL e PEPP. O Teste t-Student pareado é um teste paramétrico, que testa a igualdade das médias de uma variável cujos valores são obtidos em observações pareadas (duas amostras independentes). Para as provas PCF e PESDT foi adotado o teste de Friedman, um teste não-paramétrico, que testa a igualdade das medianas de uma variável cujos valores são obtidos em observações pareadas (amostras não independentes).

Para realizar a análise estatística pertinente, fixou-se em 0,05 ou $5 \%$ o nível de significância.

\section{RESULTADOS}

Inicialmente, apresenta-se a caracterização dos sujeitos dos grupos GA e GBRAD, quanto à idade cronológica e quociente intelectual (QI) estimado. As estatísticas descritivas média, mediana, desvio-padrão (DP), valor mínimo e valor máximo considerando-se os critérios de inclusão de idade cronológica e quociente intelectual (QI) estimado são apresentadas a seguir nas Tabelas 1 e 2 . Cabe ressaltar que não há diferença estatisticamente significante entre os dois grupos estudados GA e GBRAD quanto ao quociente intelectual estimado.

$\mathrm{Na}$ Tabela 3, são apresentados os valores de mediana, média e desvio-padrão, valor mínimo e valor máximo e p-valores calculados das respostas nas provas que compõem a avaliação da linguagem oral e escrita: PCF, TVIP, PLPP, PCL, PEPP PESDT em cada grupo estudado GA e GBRAD. 
Tabela 1 - Valores das estatísticas descritivas para a idade cronológica (em anos), nos grupos GA e GBRAD

\begin{tabular}{ccccccc}
\hline Grupo & N & Média & $\begin{array}{c}\text { Desvio } \\
\text { padrão }\end{array}$ & Mínimo & Mediana & Máximo \\
\hline GA & 22 & 16,0 & 4,4 & 10,6 & 15,5 & 27,2 \\
GBRAD & 22 & 15,9 & 4,3 & 10,9 & 15,3 & 26,7 \\
\hline
\end{tabular}

Tabela 2 - Valores das estatísticas descritivas e p-valor calculado (teste t-pareado) para o quociente intelectual (QI) estimado, nos grupos GA e GBRAD

\begin{tabular}{cccccccc}
\hline Grupo & N & Média & $\begin{array}{c}\text { Desvio } \\
\text { padrão }\end{array}$ & Mínimo & Mediana & Máximo & p-valor \\
\hline GA & 22 & 93,2 & 13,8 & 68 & 90,5 & 117 & \\
GBRAD & 22 & 100,2 & 11,1 & 75 & 103,0 & 117 & 0,089 \\
\hline
\end{tabular}

Teste t-pareado

Tabela 3 - Média, mediana e desvio-padrão (DP), valor mínimo e valor máximo e p-valores calculados das respostas nas provas que compõem a avaliação da linguagem oral e escrita

\begin{tabular}{ccccccccc}
\hline & Grupo & N & Média & DP & Mínimo & Mediana & Máximo & p-valor \\
\hline \multirow{2}{*}{ PCF } & GA & 22 & 35,6 & 4,5 & 26 & 36,5 & 40 & \\
& GBRAD & 22 & 38,6 & 2,0 & 34 & 40,0 & 40 & $0,000^{*}$ \\
\hline \multirow{2}{*}{ TVIP } & GA & 22 & 97,9 & 15,2 & 53 & 96,5 & 118 & \\
& GBRAD & 22 & 108,8 & 7,5 & 92 & 107,5 & 121 & $0,003^{*}$ \\
\hline \multirow{2}{*}{ PLPP } & GA & 22 & 86,2 & 6,0 & 61 & 88,0 & 90 & \\
& GBRAD & 22 & 87,1 & 3,1 & 78 & 88,0 & 90 & 0,504 \\
\hline \multirow{2}{*}{ PCL } & GA & 22 & 5,3 & 3,5 & 0 & 6,0 & 13 & \\
& GBRAD & 22 & 8,8 & 2,1 & 5 & 9,0 & 13 & $0,000^{*}$ \\
\hline \multirow{2}{*}{ PEPP } & GA & 22 & 57,5 & 11,8 & 18 & 60,0 & 70 & \\
& GBRAD & 22 & 60,0 & 8,0 & 45 & 63,0 & 70 & 0,312 \\
\hline \multirow{2}{*}{ PESDT } & GA & 22 & 4,1 & 1,6 & 0 & 4,0 & 7 & \\
& GBRAD & 22 & 4,6 & 1,0 & 3 & 5,0 & 7 & 0,467 \\
\hline
\end{tabular}

Teste t-pareado para as provas fonoaudiológicas: TVIP, PLPP, PCL e PEPP.

Teste de Friedman para as provas fonoaudiológicas: PCF e PESDT

A Figura 1 apresenta a distribuição dos sujeitos por grupo estudado GA e GBRAD quanto ao desempenho nas seguintes provas: PCF, TVIP, PLPP, PCL, PEPP e na PESDT.

A análise estatística revelou diferenças significantes entre as medianas da PCF, e entre as médias do TVIP e da PCL nos dois grupos estudados $(p<0,05)$. Nas demais provas não foram detectadas diferenças estatisticamente significantes entre os grupos estudados.

\section{DISCUSSÃO}

Para avaliação da linguagem oral e escrita, os testes selecionados foram: PCF, TVIP, PLPP, PCL, PEPP e PESDT.

A PCF avaliou o processamento fonológico. A consciência fonológica é um tipo de consciência metalinguística e refere-se tanto à consciência de que a fala pode ser segmentada quanto à habili- 


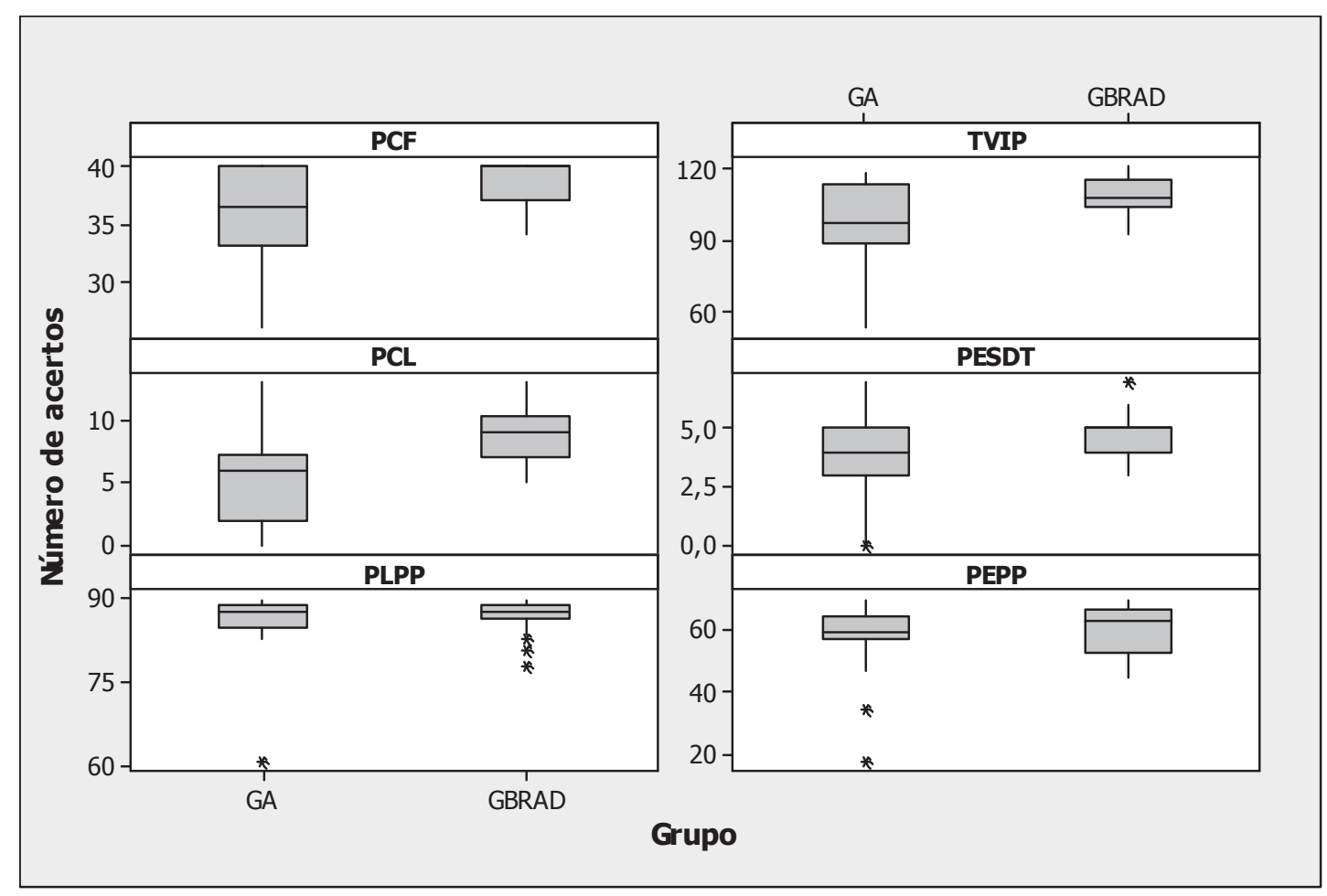

Figura 1 - Distribuição dos indivíduos por grupo estudado GA e GBRAD quanto ao desempenho na prova de consciência fonológica (PCF), no teste de vocabulário por imagem Peabody (TVIP), na prova de leitura de palavras e pseudopalavras (PLPP), na prova de compreensão de leitura (PCL), na prova de escrita sob ditado de palavras e pseudopalavras (PEPP) e na prova de escrita semidirigida de textos (PESDT)

dade de manipular intencionalmente tais segmentos ${ }^{19}$.

Os sujeitos do grupo GA obtiveram uma média de 35,6 acertos enquanto a média dos sujeitos do grupo GBRAD foi de 38,6 acertos como se pode observar na Tabela 3 e também na Figura 1. A análise estatística revelou diferença estatisticamente significante ao se comparar os dois grupos na PCF, sendo que o grupo GA apresentou um desempenho inferior ao do grupo GBRAD. A alteração no processamento fonológico manifesta-se em diversas competências linguísticas sendo que uma delas é a dificuldade de consciência fonológica.

Verificou-se que todos os sujeitos do grupo GA obtiveram bons resultados, caracterizados por desempenho igual ou superior a três pontos em cada subteste, quanto à síntese silábica, segmentação silábica e transposição silábica. Este resultado já era esperado, visto que a consciência silábica emerge em período muito precoce na vida da criança e como já havia sido verificado em estudo de sujeitos hiperléxicos com Síndrome de Asperger ${ }^{20}$.

Por outro lado, foi possível observar que as dificuldades dos sujeitos do grupo GA na prova de consciência fonológica, concentraram-se nas provas fonêmicas, visto que $45,45 \%$ do grupo apresentaram dificuldades na tarefa de segmentação fonêmica, $22,72 \%$ na tarefa de síntese fonêmica e $18,18 \%$ na tarefa de transposição fonêmica. As tarefas fonêmicas estão entre as mais difíceis de serem realizadas em sujeitos com desenvolvimento típico ${ }^{13,21}$ e também em sujeitos hiperléxicos com Síndrome de Asperger ${ }^{20}$.

Pelo fato de ter sido relatada uma variação dos resultados da prova de consciência fonológica em função do nível escolar ${ }^{13}$, torna-se importante ressaltar que todos os sujeitos do grupo GA apresentaram um mínimo de quatro anos de escolaridade, o que não impediria que os sujeitos do grupo GA apresentassem um desempenho melhor nas provas fonêmicas.

O desenvolvimento da consciência de que a fala tem uma estrutura fonêmica subjacente é essencial à aquisição da leitura e os resultados deste estudo mostraram que a habilidade de consciência fonológica encontrou-se prejudicada no grupo GA e que as tarefas fonêmicas foram as mais difíceis, o que permite concluir que a habilidade em manipular os sons do ponto de vista fonêmico parece não ter 
emergido completamente nos sujeitos do grupo GA estudados. Este dado também foi observado por outro autor ao estudar hiperléxicos com Síndrome de Asperger, na faixa etária de 15 anos ${ }^{20}$.

Há uma alta correlação entre distúrbios de leitura e escrita e alterações no processamento fonológico. Trabalhos anteriormente realizados ${ }^{22}$ sugerem como possível origem para as alterações das representações fonológicas nas crianças com dificuldade de leitura e escrita, um déficit perceptual básico que poderia explicar os distúrbios de processamento da informação linguística que, por sua vez, é formada por estímulos de curta duração, como, por exemplo, fonemas plosivos. No entanto, esta teoria parece não explicar a alteração no processamento fonológico encontrada nos sujeitos do grupo GA, pois estes sujeitos apresentaram bom desempenho em tarefas de discriminação de frequência e duração de sons, que implicam julgamentos de ordenação temporal para estímulos auditivos breves. Em contrapartida, outros modelos preconizam que as dificuldades no processamento fonológico ocorrem pela falha no desenvolvimento de competências metafonológicas importantes para o processo de aprendizagem ${ }^{23}$.

O TVIP foi utilizado para o estudo padronizado das habilidades de compreensão verbal e vem sendo utilizado para avaliar sujeitos com transtorno global do desenvolvimento ${ }^{24}$. À análise dos resultados do TVIP, verificou-se que os sujeitos do grupo GA alcançaram a média de 97,9 acertos e os do grupo GBRAD, 108,8 acertos. A análise estatística mostrou haver diferença significante entre o desempenho dos dois grupos no TVIP, com resultados piores para o grupo GA, o que indica que há uma dificuldade em relação à linguagem receptiva oral nos sujeitos do grupo GA.

Outros estudos realizados em sujeitos com autismo sem retardo mental severo associado ${ }^{25,26} \mathrm{e}$ em indivíduos com Síndrome de Asperger ${ }^{27,28}$ revelaram prejuízo de linguagem receptiva.

A utilidade do teste Peabody foi anteriormente demonstrada ${ }^{26}$ quando foi aplicado na discriminação de três grupos de sujeitos autistas, sendo um com alterações de linguagem, outro com alterações de linguagem border-line e o outro com linguagem normal. Além disso, os resultados do TVIP foram capazes de predizer o comportamento de adaptação, conforme medido pela Escala Vineland ${ }^{29}$, quanto ao aspecto comunicativo em sujeitos com Síndrome de Asperger e Autismo de alto-funcionamento. Ou seja, os resultados do TVIP foram tão piores quanto o desempenho no aspecto comunicativo da Escala Vineland desses sujeitos. Nos autistas de alto-funcionamento e nos sujeitos com Síndrome de Asperger, acredita-se que os resulta- dos dos testes que avaliam linguagem e memória sejam importantes para predizer sua capacidade de adaptação social ${ }^{29}$.

Para a avaliação da leitura foram utilizadas as provas denominadas abreviadamente PLPP e PCL. A PLPP foi utilizada com o objetivo de avaliar a decodificação da leitura e a PCL, a compreensão de leitura. Os resultados da PLPP revelaram que os sujeitos do grupo GA apresentaram, em média, 86,2 acertos e os sujeitos do grupo GBRAD apresentaram 87,1 acertos, como se pode verificar na Tabela 3 e na Figura 1. Ao comparar os grupos GA e GBRAD, não se observou diferença estatisticamente significante em relação ao desempenho na PLPP. Em relação aos resultados da PCL, verificouse que os sujeitos do grupo GA apresentaram em média, 5,3 acertos, o que se refere ao número de nodos recontados e, os sujeitos do grupo GBRAD apresentaram 8,8 acertos. Nesta prova, foi verificada diferença estatisticamente significante, ao se comparar o desempenho dos dois grupos, tendo o grupo GA apresentado desempenho inferior ao do grupo GBRAD como se pode notar na Tabela 3. Resultados semelhantes em provas de leitura e escrita foram observados em indivíduos hiperléxicos com Síndrome de Asperger ${ }^{20}$.

A leitura pressupõe a existência de dois componentes: a decodificação e a compreensão. A decodificação refere-se aos processos de reconhecimento da palavra escrita. A compreensão é definida como o processo pelo qual as palavras, sentenças ou textos são interpretados ${ }^{23}$. Sendo assim, a compreensão e o processamento de unidades linguísticas amplas como frases, enunciados e textos é uma habilidade complexa que envolve dois tipos de componentes, um específico à leitura e outro geral, inespecífico. Os processos de reconhecimento visual e decodificação das palavras isoladas são componentes específicos à leitura, e os componentes gerais inespecíficos estão relacionados à capacidade linguística de compreensão e envolvem capacidades gerais de atenção, memória, inteligência e conhecimentos gerais ${ }^{30}$. Os sujeitos do grupo GA mostraram adequação dos processos de reconhecimento visual e decodificação grafofonêmica, pois foram capazes de converter as letras em seus respectivos sons, conforme comprovado pelo desempenho adequado na PLPP e mostraram prejuízo dos processos de compreensão, inespecíficos à leitura, conforme medido pelo desempenho dos sujeitos do grupo GA na PCL.

Sugeriu-se que os sujeitos com distúrbio de leitura possam ser divididos em diferentes subgrupos, de acordo com suas habilidades de reconhecimento da palavra e de compreensão auditiva ${ }^{31}$. Um subgrupo tradicionalmente denominado como disléxico, 
teria pobre reconhecimento da palavra, mas boa compreensão. Outro grupo teria o reconhecimento da palavra e a compreensão auditiva prejudicados e um terceiro grupo seria aquele com boa decodificação, mas, em geral, com prejuízo de compreensão, que foi denominado hiperléxico ${ }^{31}$. Todos os três grupos teriam problemas de leitura, mas por diferentes razões. Vale ressaltar que embora não se tenha um teste padronizado para avaliar a presença de hiperlexia, 21 sujeitos do grupo GA apresentaram indícios de serem hiperléxicos, conforme informações colhidas no questionário realizado para obter dados sobre a história clínica dos sujeitos.

Hiperlexia é o termo utilizado para caracterizar sujeitos que apresentam uma capacidade especial para identificar palavras escritas, que emerge precocemente, anterior ao aprendizado da leitura e escrita e associada geralmente a distúrbio de linguagem ${ }^{24}$, a uma inabilidade para compreender a leitura, dificuldade de comportamento e de relacionamento pessoal ${ }^{32}$. Pesquisas anteriores ${ }^{32,33}$ verificaram que a incidência de hiperlexia foi alta em crianças com transtorno global do desenvolvimento, achado este também observado no grupo GA estudado.

Apesar da utilização de modelos que permitam a divisão dos distúrbios de leitura e escrita em subgrupos serem úteis, a noção da continuidade do espectro de manifestações dos distúrbios de linguagem escrita conforme apresentado anteriormente ${ }^{23}$ parece ser a mais adequada para o diagnóstico, prognóstico e tratamento dos distúrbios de leitura e escrita. O modelo defendido pelas autoras assume que nos distúrbios de leitura e escrita há um déficit no processamento fonológico, no entanto, há um continuum de manifestações que vão desde a dislexia pura até os distúrbios de leitura e escrita passando por todos os padrões intermediários como, por exemplo, a hiperlexia.

Outro ponto a ser discutido, refere-se ao fato de que a PCL também permite a avaliação de um componente da linguagem pragmática, ou seja, a realização de inferências. A recontagem da história "O urubu e as pombas" implica a utilização de verbos que denotam um estado mental como, por exemplo, achar e reconhecer. Verbos que denotam estado mental correspondem a uma classe de verbos que refletem um estado interno como um significado primário ${ }^{8}$. Outros exemplos de verbos que denotam estado mental são: pensar, acreditar, esquecer, gostar. Sujeitos com Síndrome de Asperger e Autismo de alto-funcionamento têm dificuldade em vários aspectos de linguagem pragmática, e isto inclui dificuldade em realizar inferências, especialmente inferências sobre estados mentais ${ }^{5-7}$.
Compreender e utilizar a linguagem envolve a decodificação do texto, mas também envolve a construção do significado por meio de uma integração entre o conhecimento do sujeito e o texto permitindo a realização de hipóteses e inferências ${ }^{24}$. Os sujeitos do grupo GA demonstraram dificuldade em compreender o texto lido e isto também reflete a dificuldade em realizar inferências sobre aquilo que os personagens da história pensaram, o que confirma a falha na "Teoria da Mente" frequentemente atribuída a sujeitos com Síndrome de Asperger e Autismo. Segundo esta teoria, sujeitos com Autismo ou Síndrome de Asperger são incapazes de atribuir estados mentais a eles mesmos e aos outros ${ }^{17,34}$.

Há indícios de que muito do desenvolvimento da linguagem relaciona-se à habilidade do falante e do ouvinte em compreender pontos de vista considerando contextos. Estudos realizados ${ }^{17,34}$ discutem a sequência da evolução das habilidades em compreender os desejos e as intenções das pessoas e mostraram que o sujeito autista tem um curso de desenvolvimento diferente do indivíduo com desenvolvimento típico.

Para a avaliação da escrita foram utilizadas as provas denominadas abreviadamente PEPP e PESDT. A PEPP foi utilizada para avaliar a correspondência fonografêmica. A PESDT foi utilizada para caracterizar a compreensão de sequência de eventos envolvendo atribuição de estados mentais e elaboração de narrativas ${ }^{24}$.

Em relação ao desempenho dos sujeitos na PEPP, verificou-se que o grupo GA apresentou média de 57,5 de acertos e o grupo GBRAD de 60 acertos. Sendo assim, pode-se afirmar que os sujeitos do grupo GA foram capazes de fazer a codificação na escrita, isto é, converter os sons da fala ouvidos em seus grafemas correspondentes. Pode-se pressupor que os sujeitos do grupo GA completaram os estágios alfabético e ortográfico de aquisição de leitura e escrita, conforme descrito anteriormente ${ }^{19}$, pois foram capazes de ler e escrever palavras reais e pseudopalavras, distribuídas em palavras irregulares, regulares e regras.

Quanto à PESDT, os sujeitos do grupo GA apresentaram média de 4,1 acertos, e o grupo GBRAD de 4,6 acertos, o que significa número de nodos recontados por meio da escrita. Não foram verificadas diferenças estatisticamente significantes entre os grupos GA e GBRAD na PESDT.

Vale lembrar que a história utilizada para a PESDT foi um tipo de história denominada comportamental, ou seja, envolve uma pessoa em ação rotineira. Verificou-se ${ }^{18}$ que crianças na faixa etária de cinco e seis anos foram capazes de ordenar temporalmente os episódios da mesma história utilizada neste estudo e que foram capazes de narrar 
as ações envolvidas nos episódios. Portanto, talvez, o tipo de história selecionada tenha sido bastante simples para os sujeitos da faixa etária avaliada neste estudo, o que favoreceu a semelhança de desempenho entre os grupos GA e GBRAD.

Além disso, conforme explicado anteriormente, a história - Comportamental II - permite narrativas do tipo causal e é uma das histórias utilizadas para avaliar a "Teoria da Mente", no entanto esta história não envolve a atribuição de intencionalidade ${ }^{17}$, o que também pode ter favorecido a semelhança de desempenho entre os grupos GA e GBRAD. Ao utilizarem a mesma sequência de figuras, outros pesquisadores ${ }^{17}$ não verificaram diferenças entre 0 desempenho de crianças autistas e de um grupo controle de crianças com desenvolvimento típico. Para este estudo, teria sido interessante também a utilização de histórias do tipo - Intencional - as quais permitem que a intencionalidade possa ser narrada e desta forma, comparar o desempenho dos grupos GA e GBRAD. Talvez, esta situação evidenciasse diferença estatisticamente significante entre os grupos GA e GBRAD.

Os distúrbios de linguagem e de comunicação estão presentes nos quadros de Autismo, conforme a própria definição do quadro no DSM-IV ${ }^{3}$ e também na Síndrome de Asperger ${ }^{20,28}$. Há um consenso geral de que estes sujeitos apresentam alterações em relação à linguagem pragmática e de discurso ${ }^{5-9,34}$. Recentemente, poucos estudos foram publicados com o objetivo de avaliar a linguagem do ponto de vista fonológico, sintático e semântico em sujeitos autistas de alto-funcionamento e com Síndrome de Asperger.

Neste estudo, de maneira geral, os sujeitos do grupo GA apresentaram alterações em nível fonológico, conforme observado pelo resultado da PCF; em nível semântico, conforme resultado no TVIP e em nível pragmático, conforme resultado obtido na PCL. Portanto, a maioria dos sujeitos do grupo GA apresentou um distúrbio de linguagem que ficou evidente em pelo menos três níveis de linguagem: fonológico, semântico e pragmático. Estes resultados corroboram os achados de outros autores que também identificaram alterações nos níveis fonológico, sintático, semântico e pragmático da linguagem $8,26,28$.

Vale ressaltar que neste estudo, não foi possível avaliar o nível sintático da linguagem de maneira explícita, pois não havia sido feito um procedimento específico para avaliar este nível de linguagem.

Outra maneira de se discutir linguagem, do ponto de vista didático e estratégico, é entendê-la como a intersecção de três componentes: forma, conteúdo e uso, sendo a integração destes três componentes essencial ${ }^{35}$. Ao tomar este referencial teórico, pode-se pressupor que os sujeitos do grupo GA apresentaram alteração de forma, conteúdo e uso da linguagem, reforçando a existência de um distúrbio de linguagem em vários níveis nestes sujeitos.

Ao analisar a linguagem oral e escrita, conforme o desempenho em cada prova, todos os sujeitos do grupo GA apresentaram desempenho inadequado em relação à linguagem, principalmente em tarefas que envolvem linguagem receptiva oral e gráfica, conforme verificado pelo desempenho no TVIP, na PCF e na PCL.

Todos os sujeitos com idade superior a dez anos que participaram deste estudo, independente da idade na qual começaram a falar, apresentaram alteração de linguagem. Portanto, parece que o comportamento de início da fala, como um dado isolado, não determina o desenvolvimento futuro da linguagem.

Com base nos resultados desta pesquisa, alguns questionamentos poderiam ser levantados. Será que os sujeitos do grupo GA poderiam ser divididos em subtipos conforme os resultados das provas utilizadas para avaliar a linguagem? $\mathrm{E}$, ainda, utilizando a mesma avaliação de linguagem deste estudo, qual seria o comportamento de sujeitos com distúrbio específico de linguagem ou com distúrbio de leitura e escrita? Outros estudos devem ser conduzidos para auxiliar na busca da causa dos transtornos globais do desenvolvimento e na caracterização do desempenho destes sujeitos quanto à linguagem, o que por sua vez, permitirá o desenvolvimento de abordagens terapêuticas mais específicas.

\section{CONCLUSÃO}

$\mathrm{Na}$ avaliação da linguagem oral e escrita, o grupo de indivíduos com Síndrome de Asperger caracterizou-se por um desempenho pior do que o grupo de indivíduos de baixo risco para alterações do desenvolvimento nas provas fonoaudiológicas de consciência fonológica, teste de vocabulário por imagem Peabody e prova de compreensão de leitura. 


\begin{abstract}
Purpose: to evaluate and characterize the oral and written language of subjects with Asperger Syndrome and compare them with a group of subjects with typical development. Methods: a total of 44 subjects were assessed and divided in two groups. The Asperger group was composed by 22 subjects diagnosed with Asperger Syndrome by an expert clinical team following the DSM-IV criteria. The comparison group, referred to as low risk for developmental disorders was also composed by 22 subjects matched with the subjects in Asperger group by chronological age. All the assessed subjects were right-handed males, with chronological ages between 10 and 30 years and intelligence quotients above 68 according to Wechsler Scale. They completed the following assessment tools: phonological conscience proof, image Peabody vocabulary test, words and pseudo words reading proof, reading comprehension proof, writing proof of dictated words and pseudo words, and the semi-directed text writing proof. Results: the statistical analysis revealed significant statistical differences between the medians obtained for the phonological conscience proof and the means of the image Peabody vocabulary test and the reading comprehension proof in both studied groups $(p<0.05)$. Conclusion: for the oral and written language assessments the Asperger Syndrome group was characterized by a worse performance then the one displayed by the group of subjects with a typical development in the phonological conscience proof, Peabody vocabulary test and the reading comprehension proof, indicating handicaps in the phonological, semantic and pragmatic language levels.
\end{abstract}

KEYWORDS: Asperger Syndrome; Language Disorders; Language Tests

\section{REFERÊNCIAS}

1. Hippler K, Klicpera C. A retrospective analysis of the clinical case records of "autistic psychopaths" diagnosed by Hans Asperger and his team at the University Children's Hospital, Vienna. Philos Trans R Soc Lond B Biol Sci. 2003; 358:291-301.

2. Wing L. Asperger's syndrome: a clinical account. Psychol Med. 1981; 11(1):115-29.

3. American Psychiatric Association. Diagnostic and statistic manual of mental disorders. 4. ed. Washington, DC: APA; 1994. 994p.

4. Organização Mundial de Saúde (OMS). Classificação de transtornos mentais e de comportamentos da CID-10: descrições clínicas e diretrizes diagnósticas. Porto Alegre: Artes Médicas; 1993. 351p.

5. Saalasti S, Lepistö T, Toppila E, Kujala T, Laakso $M$, Nieminen-von Wendt $T$, et al. Language abilities of children with Asperger syndrome. J Autism Dev Disord. 2008; 38(8):1574-80. Epub 2008 Mar 7.

6. Woodbury-Smith MR, Volkmar FR. Asperger syndrome. Eur Child Adolesc Psychiatr. 2008, Jun 18 [Epub ahead of print]

7. Beaumont R, Newcombe P. Theory of mind and central coherence in adults with high-functioning autism or Asperger syndrome. Autism. 2006; 10(4):365-82.

8. Ramberg C, Ehlers S, Nydén A, Johansson M, Gillberg C. Language and pragmatic functions in school-aged children on the autism spectrum. Eur $\mathrm{J}$ Disord Commun. 1996; 31(4):387-413.
9. Kaland N, Callesen K, MØller-Nielsen A, Mortensen EL, Smith L. Performance of children and adolescents with Asperger syndrome or highfunctioning autism on advanced theory of mind tasks. J Autism Dev Disord. 2008; 38(6):1112-23.

10. Spreen O, Strauss E. A compendium of neuropsychological tests: administration, norms and commentary. New York: Oxford University Press; 1991. 736p.

11. Oldfield RC. The assessment and analysis of handedness: the Edinburgh inventory. Neuropsychol. 1971; 9(1):97-113.

12. Silman S, Silverman CA. Auditory diagnosis. San Diego: Academic Press; 1991. 412p.

13. Capovilla AGS, Capovilla FC. Prova de consciência fonológica: desenvolvimento de dez habilidades da pré-escola à segunda série. Temas Desenv. 1998; 7(37):14-20.

14. Capovilla F, Nunes LROP, Nogueira D, Nunes $D$, Araújo I, Bernat AB, et al. Desenvolvimento do vocabulário receptivo auditivo da pré-escola à oitava série: normatização fluminense baseada em aplicação coletiva da tradução brasileira do Peabody Picture Vocabulary Test. Ciência Cognitiva: Teoria, Pesquisa e Aplicação. 1997; 1(1):381-440.

15. Capovilla AGS, Capovilla FC. Problemas de leitura e escrita: como identificar, prevenir e remediar numa abordagem fônica. São Paulo: Memnon; 2000. 251p. 
16. Moraes ZR. Estilos de linguagem como facilitadores da memória [tese]. São Paulo (SP): Universidade Federal de São Paulo; 1999.

17. Baron-Cohen S, Leslie AM, Frith U. Mechanical, behavioral and intentional understanding of pictures in autistic children. Brit J Dev Psychol. 1986; 4:113-25.

18. Artoni AL. Caracterização de narrativas de crianças de 5 e 6 anos: correlações entre episódios e ações [dissertação]. São Paulo (SP): Universidade Federal de São Paulo; 2001.

19. Capovilla AGS, Capovilla, FC. Alfabetização: método fônico. São Paulo: Memnon; 2002. 393 p.

20. Silva RCD. Hiperléxicos com transtorno de Asperger. Caracterização da leitura e escrita de textos e palavras [dissertação]. São Paulo (SP): Universidade Federal de São Paulo; 2004.

21. Santos MTM, Pereira LD. Consciência fonológica. In: Pereira LD, Schochat E. Processamento auditivo central manual de avaliação. São Paulo: Lovise; 1997. p.187-96.

22. Gaab N, Gabrieli JD, Deutsch GK, TallalP, Temple E. Neural correlates of rapid auditory processing are disrupted in children with developmental dyslexia and ameliorated with training: an fMRI study. Restor Neurol Neurosci. 2007; 25(3-4):295-310.

23. Navas ALGP, Santos MTM. Distúrbios de leitura e escrita: teoria e prática. Barueri: Manole; 2002. $389 \mathrm{p}$.

24. Condouris K, Meyer E, Tager-Flusberg H. The relationship between standardized measures of language and measures of spontaneous speech in children with autism. Am J Speech Lang Pathol. 2003; 12(3):349-58.

25. Rapin I, Dunn M. Update on language disorders of individuals on the autistic spectrum. Brain Dev. 2003; 25(3):166-72.
26. Tager-Flusberg $H$, Joseph RM. Identifying neurocognitive phenotypes in autism. Philos Trans R Soc Lond B Biol Sci. 2003; 358(1430):303-14.

27. Colle L, Baron-Cohen S, Wheelwright S, Van der Lely HK. Narrative discourse in adults with highfunctioning autism or Asperger syndrome. J Autism Dev Disord. 2008; 38(1):28-40.

28. Howlin P. Outcome in high-functioning adults with autism with and without early language delays: implications for the differentiation between autism and Asperger Syndrome. J Autism Dev Disord. 2003; 33(1):3-13.

29. Liss $M$, Harel B, Fein D, Allen D, Dunn M, Feinstein C, et al. Predictors and correlates of adaptative functioning in children with developmental disorders. J Autism Dev Disord. 2001; 31(2):219-30. 30. Fodor J. Modularity of mind. Cambridge: MIT Press; 1983.164 p.

31. Nation K, Clarke P, Wright B, Williams C. Patterns of reading ability in children with autism spectrum disorder. J Autism Dev Disord.2006; 36(7):911-9.

32. Grigorenko EL, Klin A, Volkmar F. Annotation: Hyperlexia: disability or superability? J Child Psychol Psychiatry. 2003; 44(8):1079-91.

33. Newman TM, Macomber D, Naples AJ, Babitz $\mathrm{T}$, Volkmar F, Grigorenko EL. Hyperlexia in children with autism spectrum disorders. J Autism Dev Disord. 2007; 37(4):760-74.

34. Duverger H, Da Fonseca D, Bailly D, Deruelle C. [Theory of mind in Asperger syndrome]. Encephale. 2007; 33(4 Pt 1):592-7.

35. Brinton B, Fujiki, M. Conversational management: introduction and overview. In: Brinton B, Fujiki $M$. Conversational management with languageimpaired children: pragmatic assessment and intervention. Rockville, Maryland: Aspen; 1989. (Excellence in Practice Series). p 1-19.

DOI: 10.1590 / S1516-18462009005000004

RECEBIDO EM: 04/12/2007

ACEITO EM: 03/06/2008

Endereço para correspondência:

Karin Ziliotto Dias

Av. Cotovia, 80 ap. 141

São Paulo - SP

CEP: 04517-000

E-mail: rdkz@uol.com.br 